\title{
ATENDIMENTO AOS ESTUDANTES COM NECESSIDADES EDUCACIONAIS ESPECÍFICAS EM UNIVERSIDADES E INSTITUTOS FEDERAIS DO NORDESTE: ANÁLISE DOS SITES INSTITUCIONAIS
}

http://dx.doi.org/10.5902/2318133847107

\author{
Thales Fabricio da Costa e Silva ${ }^{1}$ \\ Luana Ugalde da Costa² \\ Débora Bruna Alves Almeida ${ }^{3}$ \\ Leandro Campi Prearo 4
}

\begin{abstract}
Resumo
Neste estudo apresenta-se resultados de análise da acessibilidade às informações sobre os setores de atendimento aos estudantes com necessidades educacionais específicas nos sites das universidades e institutos federais de educação, ciência e tecnologia do Nordeste. A partir de uma pesquisa documental, foram levantados dados de 29 sites, analisando-os a partir da estatística descritiva e análise de clusters. Os resultados apontaram que, mesmo não havendo um padrão de informações disponíveis nos sites pesquisados, é possível definir as instituições com maior e menor acessibilidade às informações sobre estes setores. A pesquisa reforça a acessibilidade nos sites institucionais como uma estratégia de superação das barreiras à inclusão, especialmente as barreiras tecnológicas, atitudinais e comunicacionais.

Palavras-chave: acessibilidade; inclusão; acesso à informação.
\end{abstract}

\section{ASSISTANCE TO STUDENTS WITH SPECIFIC EDUCATIONAL NEEDS AT UNIVERSITIES AND FEDERAL INSTITUTES OF THE NORTHEAST: ANALYSIS OF INSTITUTIONAL SITES}

\begin{abstract}
This study analyzes the accessibility to information about the sectors of assistance to students with specific educational needs on the websites of the universities and federal institutes of education, science and technology of the Northeast. From a documentary research, data were collected in 29 places, analyzing them based on descriptive statistics and cluster analysis. The results showed that, although there is no standard of information available on the researched sites, it is possible to define institutions with greater and lesser accessibility to information in these sectors. The research reinforces accessibility on institutional websites as a strategy to overcome barriers to inclusion, especially technological, attitudinal and communicational barriers.

Key-words: accessibility; inclusion; access to information.
\end{abstract}

\footnotetext{
${ }^{1}$ Universidade Municipal de São Caetano do Sul, Brasil. E-mail: thalespsic@gmail.com.

2 Instituto Federal de Educação, Ciência e Tecnologia do Acre, Brasil. E-mail: luana.costa@ifac.edu.br.

3 Universidade Municipal de São Caetano do Sul, Brasil. E-mail: debora.almeida@ifce.edu.br.

4 Universidade Municipal de São Caetano do Sul, Brasil. E-mail: leandro.prearo@gmail.com.

Regae: Rev. Gest Aval. Educ. 


\section{Introdução}

A s inúmeras legislações nacionais e produções científicas envolvendo pessoas com deficiência na sociedade brasileira direcionam a uma condição inquestionável: a inclusão deve ser uma prática constante em todos os espaços de ocupação humana. Entre esses destacam-se os ambientes educacionais, nos quais a inclusão é um direito reconhecido pela Constituição Federal de 1988, que assevera o dever do Estado de ofertar o atendimento educacional especializado - AEE às pessoas com deficiência, preferencialmente na rede regular de ensino (Brasil, 1988).

Tem-se assistido um aumento de estudantes com necessidades educacionais específicas no ambiente escolar, especialmente no contexto universitário, resultado das ações afirmativas para pessoas com deficiência adotadas no país, embasadas em discussões de âmbito nacional e internacional que contribuíram para o subsídio de provisões institucionais voltadas não somente ao ingresso, mas ao apoio e acompanhamento dessa população, com a finalidade de prover sua permanência e sucesso acadêmico (Cabral, 2018).

Uma das estratégias desenvolvidas no país visando à inclusão dos estudantes com deficiência nas modalidades de ensino profissional, tecnológico e superior foi a criação de programas para $o$ atendimento a estudantes com NEE - que englobam estudantes com deficiência, transtornos globais do desenvolvimento ou altas habilidades ou superdotação (Brasil, 2011a). Entre esses destacam-se o Programa TEC NEP: educação, tecnologia e profissionalização para pessoas com NEE, e o Programa Incluir: acessibilidade na educação superior. O primeiro, implantado em 2000 na rede federal de educação profissional, científica e tecnológica, impulsionou a criação dos Núcleos de Atendimento às Pessoas com Necessidades Específicas - Napne (Costa, 2018), e o segundo, criado em 2005, tinha o objetivo de fomentar a criação e consolidação de núcleos de acessibilidade nas universidades federais e, embora extinto em 2010, a assistência a esses núcleos foi continuada, a partir de 2011, pelo Plano viver sem limites (Nogueira e Oliver, 2018; Brasil, 2011b; 2010).

Para Melo e Araújo (2018) o alvo crítico em um trabalho com estudantes com NEE diz respeito ao suporte educacional, monitoramento e acompanhamento da trajetória deles em todo o percurso acadêmico por meio de uma equipe interdisciplinar, o que ainda não é a realidade de muitos desses setores nas instituições federais de educação superior - Ifes. Soma-se a isso a ausência de infraestrutura adequada ao atendimento, que envolve, além da perspectiva arquitetônica, os serviços e produtos disponibilizados às pessoas com deficiência. Essa problemática se torna mais importante quando são realizados recortes regionais e analisadas as suas particularidades sociodemográficas. A região Nordeste, por exemplo, é a região que possui as maiores taxas de deficiências física e intelectual do país e a segunda maior taxa de deficiências auditiva e visual (IBGE, 2015).

Neste cenário investigou-se como as organizações e os setores destinados ao atendimento a estudantes com NEE da região Nordeste estão articulados na prestação de serviços a esse público, sendo a consulta ao site institucional um dos meios de acessar essas informações. 
Ferreira, Chauvel e Ferreira (2006) assinalam que há muitos anos as organizações públicas brasileiras têm o desafio de administrar e projetar sites em conformidade com as diretrizes nacionais e internacionais para a acessibilidade com o intuito de se tornarem atraentes e fáceis de usar. Porém, investigações em sites nacionais têm demonstrado um baixo nível de acessibilidade, seja pela ausência ou pela presença de poucos recursos e ferramentas virtuais para minimizar os efeitos da deficiência, revelando um desafio ainda presente para 0 acesso informacional às pessoas com deficiência (Lemos-Santos, Teixeira, Kafure, 2019; Arenhardt et al., 2017; Freitas et al., 2016; Campos, Sanchez; Sousa, 2013; Melo, Silveira, Ferreira, 2013; Gurgel, Medeiros, Moura, 2012; Ferreira, Cianconi, 2011; Bach, Ferreira, Silveira, 2009; Ferreira, Santos, Silveira, 2007).

Esta produção se baseia na acessibilidade à informação para além da adoção de ferramentas para o site - como ampliação da fonte textual, cabeçalho indicativo, adoção de alto contraste e atalhos de navegação -, compreendendo, portanto, o acesso ao conteúdo de interesse da comunidade e a sua disposição nos sites institucionais. Assim, orientou-se pela seguinte problemática: os sites das Ifes do Nordeste são acessíveis quanto à prestação das informações sobre os setores de atendimento aos estudantes com NEE?

O objetivo foi o de analisar a acessibilidade às informações sobre os setores de atendimento aos estudantes com NEE nos sites das Ifes do Nordeste. Para tanto, buscouse identificar as Ifes e seus respectivos setores que ofertam serviços de acessibilidade e inclusão aos estudantes com NEE e avaliar o acesso às informações sobre estes setores disponíveis nos sites institucionais.

Por meio de uma pesquisa documental foram coletados dados em 29 sites das Ifes do Nordeste, tendo como base a presença ou ausência de treze variáveis relacionadas às informações sobre os setores de atendimento aos estudantes com NEE contidas nos sites institucionais. Os dados foram analisados por meio de estatística descritiva, usando a técnica análise de conglomerados. Os resultados apontam que, mesmo não existindo um perfil de informações disponíveis nesses sites, é possível distribuir as Ifes com maior e Ifes com menor acessibilidade às informações sobre esses setores.

\section{Informação, acessibilidade e inclusão nos sites das Ifes}

O Censo da Educação Superior 2018 registrou mais um ano de crescimento no número de matrículas no ensino superior brasileiro, totalizando 8.450 .755 estudantes matriculados, distribuídos entre 2.537 instituições públicas e privadas. Nesse cenário as Ifes são representadas por 69 universidades federais e 40 institutos federais de educação, ciência e tecnologia, ocupando lugar de destaque, pois são nas universidades federais que se encontra a maior quantidade de matrículas entre as universidades públicas e nos institutos federais que se registra a maior variação positiva de matrículas $(348,6 \%)$ nos últimos 10 anos (Brasil, 2019).

Este cenário ilustra a importância desempenhada pelas Ifes na expansão e efetivação do direito à educação superior no Brasil, processo que foi potencializado após a promulgação da lei n. 12.711/2012 e do decreto n. 9.034/2017, garantindo a reserva de, no mínimo, $50 \%$ das vagas ofertadas para estudantes que tenham cursado integralmente o ensino médio em escolas públicas e, destas, são reservadas $50 \%$ para estudantes 
oriundos de famílias com renda igual ou inferior a 1,5 salário mínimo per capita; soma-se a esta reserva o preenchimento de vagas por negros, pardos, indígenas e pessoas com deficiência, respeitando a proporção desta população no país (Brasil, 2012; 2017).

$O$ acesso de estudantes com deficiência no ensino superior os retira do campo da invisibilidade, oportuniza um processo reflexivo para todos os atores educacionais, assim como a atuação dos setores destinados especificamente aos estudantes com NEE auxilia no desenvolvimento acadêmico e no rompimento do processo discriminatório que cerca as pessoas com deficiência no percurso escolar (Lopes et al., 2016; Melo e Araújo, 2018).

Vale considerar, no entanto, que o processo de inclusão educacional no Brasil tem se constituído lentamente, enfrentando múltiplos desafios. Um destes é a mudança necessária no contexto universitário que, segundo Almeida, Bellosi e Ferreira (2015), é caracterizado por uma estrutura arquitetônica, atitudinal e pedagógica feita para um padrão de aluno proveniente das elites e dotado de condições físicas e intelectuais tidas como norma, excluindo pessoas que fujam desse perfil, como as que possuem deficiência. Ainda sobre os desafios a serem ultrapassados Nogueira e Oliver (2018) incluem a revisão do material didático, das estratégias metodológicas operadas por professores, da participação dos estudantes em atividades da vida universitária para além da sala de aula e dos processos comunicacionais.

Essas mudanças são estruturais e podem ser auxiliadas pelos setores que assistem aos estudantes com NEE. Segundo Maciel e Anache (2017) os "núcleos de acessibilidade respondem pela organização de ações institucionais que garantam a integração de pessoas com deficiência à vida acadêmica, eliminando barreiras comportamentais, pedagógicas, arquitetônicas e de comunicação" (p. 73). Quanto aos Napnes, termo original proposto pelo Programa TEC NEP não há, na literatura, nem nas legislações, um conceito conclusivo a respeito da sua natureza, embora seja correta a ideia de atendimento a pessoas com NEE que constitui a sua sigla. Rocha (2016) identificou oito nomenclaturas deste setor nos institutos federais país e Mendes (2017) problematiza que as diferenças entre as nomenclaturas remetem à existência de diferentes concepções de atendimento na Rede Federal.

Soma-se ao exposto o fato de que a implantação desses setores não ocorre de maneira uniforme. Ao tomar como exemplo a Universidade Federal do Rio Grande do Norte - UFRN - e a Universidade Federal de Campina Grande - UFCG -, ambas localizadas do nordeste brasileiro, a primeira implantou seu núcleo de acessibilidade em 2010 (UFRN, 2010) e a segunda em 2016 (UFCG, 2016), demarcando um espaço temporal significativo entre duas instituições da mesma natureza.

Um aspecto fundamental para o conhecimento da instituição é a disposição das informações acerca da infraestrutura e dos serviços ofertados à comunidade, sendo o site institucional um dos meios para acessar essas informações. Bergamo et al. (2010) identificaram 12 fatores que influenciam a escolha por uma instituição de ensino superior, destacando, entre eles, a imagem e a estrutura, transmitidas, entre outros meios, pelo site institucional. Assim, é possível considerar que, a partir das informações dispostas no seu site, uma instituição pode ser preterida em virtude de outra, especialmente por públicos que demandam serviços específicos, como é o caso das pessoas com NEE. 
Neste sentido, ao discutir o acesso aos sites de instituições educacionais, Santos et al. (2015) apontam a necessidade de ser organizado um sistema de informações acessíveis aos estudantes com NEE, especialmente que oportunizem o conhecimento prévio acerca dos apoios oferecidos pela instituição, o que constitui um fator fundamental para a adaptação desse estudante no ensino superior. Essa assertiva dialoga com a necessidade de superação das barreiras comunicacionais, apontadas pela Lei Brasileira da Inclusão (Brasil, 2015) como sendo qualquer entrave, obstáculo, atitude ou comportamento que dificulte ou impossibilite a expressão ou o recebimento de mensagens e de informações por intermédio de sistemas de comunicação e de tecnologia da informação.

A disposição dessas informações deve coadunar com a ideia de acessibilidade digital, que respalda um conceito de acessibilidade que não se resume à estrutura física, mas que está presente em todas as instâncias da vida (Freitas et al., 2016) e que atende às distintas formas de interação das pessoas com a informação, respeitando preferências e limitações - tanto de equipamentos quanto às orgânicas (Torres, Mazzoni, Alves, 2002).

\section{Metodologia}

A investigação proposta neste estudo permeou o campo da acessibilidade à informação sobre serviços prestados pelos setores que atendem estudantes com NEE nas Ifes do Nordeste. Os procedimentos metodológicos seguiram quatro etapas: a) pesquisa bibliográfica; b) pesquisa documental; c) análise dos dados; d) interpretação dos dados.

Neste estudo os documentos consultados foram os sites das Ifes do Nordeste, localizadas nos estados de Alagoas, Bahia, Ceará, Maranhão, Paraíba, Pernambuco, Piauí, Rio Grande do Norte e Sergipe. No quadro 1 apresenta-se a lista de todas as Ifes pesquisadas, totalizando 29 instituições.

Quadro 1 -

Distribuição das Ifes do Nordeste por Unidade da Federação.

\begin{tabular}{|c|c|c|}
\hline UF & Instituto federal & Universidade federal \\
\hline$\overline{A L}$ & If de Alagoas - Ifa & Uf de Alagoas - Ufal \\
\hline BA & $\begin{array}{l}\text { If Baiano - Ifbaiano } \\
\text { If da Bahia - IFBA }\end{array}$ & $\begin{array}{c}\text { Uf da Bahia - UFBA } \\
\text { Uf do Oeste da Bahia - Ufob } \\
\text { Uf do Recôncavo da Bahia - UFRB } \\
\text { Uf do Sul da Bahia - UFSB }\end{array}$ \\
\hline CE & If do Ceará - IFCE & $\begin{array}{c}\text { Uf da Integração Internacional da } \\
\text { Lusofonia Afro-Brasileira - Unilab } \\
\text { Uf do Cariri - UFCA } \\
\text { Uf do Ceará - UFC }\end{array}$ \\
\hline MA & If do Maranhão - Ifma & Uf do Maranhão - UfMA \\
\hline PB & If da Paraíba - IFPB & $\begin{array}{c}\text { Uf da Paraíba - UFPB } \\
\text { Uf de Campina Grande - UFCG }\end{array}$ \\
\hline PE & $\begin{array}{c}\text { If de Pernambuco - IFPE } \\
\text { If do Sertão Pernambucano -If } \\
\text { Sertão }\end{array}$ & $\begin{array}{l}\text { Uf de Pernambuco - UFPE } \\
\text { Uf do Vale do São Francisco - Univasf } \\
\text { Uf Rural de Pernambuco - UFRPE }\end{array}$ \\
\hline $\mathrm{PI}$ & If Do Piauí - IFPI & Uf do Piauí - UFPI \\
\hline
\end{tabular}




\begin{tabular}{|c|c|c|}
\hline RN & If do Rio Grande do Norte - IFRN & $\begin{array}{c}\text { Uf do Rio Grande do Norte - UFRN } \\
\text { Uf Rural do Semi-Árido - Ufersa }\end{array}$ \\
\hline SE & If de Sergipe - IFS & Uf de Sergipe - UFS \\
\hline
\end{tabular}

Fonte: autores.

Os dados sobre acessibilidade à informação foram coletados com base em um formulário contendo treze variáveis de verificação: 1) link sobre o setor de acessibilidade e inclusão na primeira página do site; 2) informação sobre o setor que atende ao estudante com NEE; 3) página específica para o setor; 4) serviços ofertados pelo setor; 5) informação sobre o profissional responsável pelo setor; 6) informação sobre a equipe que compõe o setor; 7) informações sobre direitos dos estudantes atendidos pelo setor; 8) público-alvo; 9) projetos e/ou programas destinados a pessoas com NEE desenvolvidos pelo setor ou em parceria; 10) formas de contato: podendo incluir endereço, e-mail, telefone ou redes sociais; 11) links para documentos importantes sobre o tema; 12) links ou arquivo dispondo a resolução de criação ou o regimento do setor; e 13) notícia recente de ações envolvendo estudantes com NEE.

As variáveis foram elaboradas considerando os conteúdos apresentados pelos sites das Ifes, porém, algumas foram referenciadas pelas Diretrizes de acessibilidade para conteúdo web (W3C, 2014), mais especificamente a diretriz 2.4 - Navegável: fornecer formas de ajudar os utilizadores a navegar, localizar conteúdos e determinar o local onde estão, como é o caso das variáveis 1, 2, 3, 11 e 12.

Para a análise dos dados, adotou-se o critério avaliativo de atribuição dos conceitos 'sim' ou 'não' às variáveis observadas em cada site pesquisado. As respostas 'não' e 'sim, de natureza binária, foram codificados em 0 e 1, respectivamente. Embora esse trabalho não colete números de forma direta, a quantificação dos resultados é possível pela técnica de transformação dos dados qualitativos observados. Essa técnica, apontada por Creswell (2007) como a transformação de dados, possibilita a transformação de dados qualitativos em quantitativos - e também o caminho inverso -, permitindo ao pesquisador comparar resultados quantitativos com dados qualitativos. Assim, o olhar qualitativo do pesquisador sobre os sites possibilitou a quantificação da acessibilidade às informações sobre os setores das Ifes.

A análise de conglomerados ou clusters analyzsis, segundo Bem, Giacomini e Waismann (2015), é uma técnica multivariada de classificação que objetiva agrupar dados de acordo com as similaridades entre eles, agrupando um conjunto de dados heterogêneos em grupos com homogeneidade. Para guiar esta aplicação, algumas premissas devem ser respeitadas, sendo uma delas o número da amostra; como, neste estudo, foram obtidas 29 amostras, o método hierárquico é o mais indicado.

\section{Apresentação dos resultados}

Durante a pesquisa documental, todas as 29 Ifes do Nordeste estavam com seus sites institucionais em pleno funcionamento. O acesso foi feito pelos pesquisadores, alimentando um banco de dados a cada variável que era observada no site. É indispensável ressaltar que esta etapa exigia o olhar qualitativo dos pesquisadores e foi a mais minuciosa, considerando que era preciso verificar todas as abas disponíveis, links, 
entre outras ferramentas disponíveis. Além disso, para ter certificação da ausência ou presença de determinada informação, foi utilizada a ferramenta de pesquisa no próprio site com os seguintes descritores: acessibilidade, inclusão, deficiência e NEE.

Entre todos os sites apenas o site da UFBA não apresentou nenhuma variável, no entanto, ao utilizar a ferramenta de pesquisa do próprio site, foram identificadas várias notícias envolvendo o tema acessibilidade e inclusão, o que gerou questionamentos nos pesquisadores acerca da ausência de informações sobre o setor em estudo. Assim, foi feita uma pesquisa no Google e foi identificado que a UFBA disponibiliza um site independente para o seu Núcleo de Apoio à Inclusão do Aluno com NEE - Nape. Embora esse dado seja significativo para o acesso à informação ele revela um ponto crítico, já que o site principal da instituição não faz referência ao Nape e a este site especificamente. Assim, para fins desta pesquisa, foram considerados os dados obtidos no site principal da UFBA.

Com o banco de dados construído a partir das 13 variáveis observadas e, considerando a variável '2' - a que mais se verificou no universo pesquisado -, identificouse que $86,2 \%(n=25)$ das Ifes dispõem algum tipo de informação sobre os setores de atendimento ao estudante com NEE, no entanto, entre estas, apenas $36 \%(n=9)$ disponibilizam link direto ao setor já na primeira página; as demais disponibilizam essa informação de forma distribuída no conteúdo de diversas páginas, como ensino ou extensão, e que não fazem menção diretamente a este tipo de atividade.

A presença de um link de acesso ao setor já na página inicial é a variável mais importante, pois facilita o acesso à informação a qualquer pessoa alheia à comunidade acadêmica e ao seu funcionamento, sobretudo ao estudante com algum tipo de limitação física, visual, auditiva ou cognitiva, permitindo o conhecimento integral sobre a instituição e os serviços do seu interesse. Essa observação se apoia na superação das barreiras nas comunicações e na informação da LBI (Brasil, 2015) e nas Diretrizes de acessibilidade para conteúdo web (W3C, 2014), que destaca a importância dos sites fornecerem formas de ajudar o usuário a navegar, localizar conteúdos e determinar o local onde ficam, como localização da página e páginas com títulos que descrevem a sua finalidade.

Quanto à variável menos observada entre as Ifes pesquisadas foi identificado que apenas $20,6 \%(n=6)$ dos sites trazem notícias recentes envolvendo estudantes com NEE. Esta variável foi escolhida para a coleta de dados porque as notícias veiculadas pela instituição ilustram o cenário de oportunidades entre ações, projetos e programas voltados ao público com NEE, bem como o engajamento deste público nas diversas atividades de ensino, pesquisa e extensão. Para a identificação desta variável, além da leitura da página inicial e de notícias, também foi utilizada a ferramenta de pesquisa no próprio site com os seguintes descritores: acessibilidade, inclusão, deficiência e NEE.

Esta publicidade dada aos diversos públicos que transitam no ensino superior é, além de um convite a habitar uma instituição inclusiva, uma estratégia de enfrentamento ao que Pereira (2008) chamou de preconceito manifestado pelo silêncio, na não manifestação, na ignorância e, sobretudo, na indiferença. Somado a isso publicizar o processo de inclusão dos estudantes com NEE é uma maneira de mitigar as barreiras atitudinais, conceituadas pela LBI (Brasil, 2015) como as atitudes ou comportamentos que prejudiquem ou impeçam a participação social da pessoa com deficiência em igualdade de condições e oportunidades com as demais pessoas. 
Com a aplicação da análise de conglomerados obteve-se o agrupamento de Ifes em dois clusters: um com 12 e outro com 17 instituições. Como a técnica objetiva agrupar elementos com a maior homogeneidade possível entre os seus elementos e diferenciar um grupo do outro a partir da maior heterogeneidade possível, os grupos obtidos foram nomeados em Ifes com maior e menor acessibilidade - considerando esta acessibilidade às informações dos setores que atuam com estudantes com NEE, conforme se discute ao longo de todo o trabalho. Os grupos e suas respectivas instituições estão detalhados no quadro 2.

Quadro 2 -

Divisão das instituições com maior e menor acessibilidade.

\begin{tabular}{|c|c|}
\hline IFES com maior acessibilidade & IFES com menor acessibilidade \\
\hline $\begin{array}{c}\text { IFCE, IFPB, IFS, Ufal, UFC, UFCA, Ufersa, } \\
\text { Ufob, UFPB, UFPE, UFPI, UFRB, UFRN, } \\
\text { UFRPE, UFS, UFSB, Univasf. }\end{array}$ & $\begin{array}{c}\text { Ifal, IFBA, IFBaiano, IFMA, IFPE, IFPI, IFRN, } \\
\text { IFSertão, UFBA, UFCG, UFMA, Unilab. }\end{array}$ \\
\hline
\end{tabular}

Fonte: autores.

A técnica utilizada permite destacar quais variáveis apresentam maior significância para a discriminação dos grupos, ou seja, quais delas influenciam a alocação de uma Ifes no grupo de instituições com maior ou menor acessibilidade às informações prestadas. Entre as variáveis observadas apenas duas não apresentaram significância para a discriminação: a 12 - presença de link ou arquivo contendo a resolução ou o regimento do setor - e a 13 - presença de notícias recentes de ações envolvendo estudantes com NEE. Vale considerar que, embora sejam elementos também importantes para garantir a acessibilidade à informação para este público, essas variáveis não são determinantes, perante a amostra, para a discriminação das instituições nos dois grupos.

Pelos dados coletados é possível verificar que, entre as Ifes com maior acessibilidade, todas disponibilizavam informações sobre os setores que atendem pessoas com NEE em algum lugar do site e, ao mesmo tempo, disponibilizavam uma página específica no site com informações do setor. Vale considerar, porém, que essas páginas também estavam distribuídas em contextos de trabalho diferentes, como assistência estudantil, extensão ou ensino, o que aponta para a indefinição do lugar ocupado pelas práticas institucionais de acessibilidade e inclusão, o que se equipara às inúmeras nomenclaturas dadas a esses setores, como foi identificado por Rocha (2016), e às diversas concepções de atendimento, como destacou Mendes (2017).

Ao comparar as instituições com maior e menor acessibilidade às informações disponíveis em seus sites institucionais, verificou-se que a Ufersa e a UFC se posicionam como as mais acessíveis e a UFBA e a UFCG como as menos acessíveis da região. As duas primeiras figuram como as mais acessíveis porque apresentam em seu site a maior quantidade de variáveis: no site da Ufersa todas as variáveis foram observadas e no da UFC apenas uma delas não foi identificada. Por sua vez, a UFCG e a UFBA estão nas últimas posições porque apresentam o menor número de variáveis observadas, sendo a UFBA nenhuma variável e a UFCG apenas a variável '12 - presença de link ou arquivo contendo a resolução ou o regimento do setor'; além disso, como já foi citado, esta variável não apresenta significância para a discriminação dos grupos. 
Vale salientar que essa classificação entre mais e menos acessíveis não representa a atuação dessas instituições quanto às demandas de estudantes com NEE, mas uma referência estrita à avaliação das informações disponíveis ao público que acessa aos seus sites.

Destaca-se que a maioria das Ifes investigadas disponibiliza sites específicos para cada campi que compõe a sua estrutura organizacional, mas como não foram documentos considerados para esta pesquisa a avaliação se baseia no site principal da instituição. Deste modo, é necessário que as Ifes compreendam que a comunidade externa ao mundo acadêmico não conhece sua estrutura institucional e a divisão de suas fontes de informação, sendo significativa a alocação do maior número de informações no seu site principal para que supere a barreira comunicacional ainda existente em alguns sites. Somado a isso, e considerando a significância das variáveis apresentadas, sugerese que cada Ifes disponibilize todas as informações que foram investigadas nesse trabalho.

Nota-se que a acessibilidade nos sites institucionais é uma estratégia de superação das inúmeras barreiras à inclusão, especialmente as barreiras tecnológicas, atitudinais e nas comunicações e na informação, o que reforça o direito da pessoa com deficiência, transtornos globais do desenvolvimento e altas habilidades/superdotação de acessar e permanecer no ambiente educacional presencial e virtual.

\section{Conclusão}

A pesquisa teve como objetivo analisar a acessibilidade às informações sobre os setores de atendimento aos estudantes com NEE nos sites das Ifes do Nordeste. A partir da pesquisa documental aos sites das 29 Ifes da região, foram levantados dados acerca da presença ou ausência de treze variáveis relacionadas às informações sobre esses setores contidas nos sites institucionais.

Foi possível identificar uma variedade de perfis, visto que as instituições não apresentaram regularidade quanto à presença ou ausência das variáveis investigadas. No entanto, foi possível perceber, a partir da análise de conglomerados, o grupo de Ifes com maior acessibilidade que - entre outras variáveis, disponibilizavam informações sobre os setores que atendem pessoas com NEE em algum lugar do site e, ao mesmo tempo, disponibilizavam uma página específica no site com informações específicas deste setor e o grupo de Ifes com menor acessibilidade - que apresentaram o menor número de variáveis - às informações dispostas sobre os setores de atendimento ao estudante com NEE. Entre essas a Ufersa e a UFC se destacam como as de maior acessibilidade e a UFBA e a UFCG como as de menor acessibilidade.

Ressalta-se que o resultado desta pesquisa não representa a atuação destas instituições frente às demandas dos estudantes com NEE, mas demonstra a necessidade dessas Ifes alimentarem seus sites institucionais com informações necessárias ao pleno atendimento dos interesses da comunidade discente, especialmente dos estudantes que possuem alguma deficiência, transtornos global do desenvolvimento ou altas habilidades/superdotação. $O$ atendimento a este aspecto faz com que as Ifes se tornem organizações mais democráticas e inclusivas, contribuindo para a mitigação das desigualdades e do processo excludente que grupos minoritários - como o das pessoas com deficiência e outras NEE - enfrentam historicamente. 
Esta pesquisa foi limitada na região Nordeste e avaliou apenas os sites principais de cada Ifes, visto que a maioria delas possui sites próprios para cada campus. No entanto, tais limitações não minimizam a importância deste estudo, considerando o perfil da região quanto à incidência de pessoas com deficiência e que os sites principais das Ifes se constituem a porta de entrada do cidadão comum às informações sobre aquela instituição. Para superar tais limitações, sugere-se que novos estudos sejam realizados, ampliando a análise para os sites das Ifes de outras regiões do país.

\section{Referências}

ALMEIDA, José Guilherme de Andrade; BELLOSI, Tereza Cristina; FERREIRA, Eliana Lúcia. Evolução da matrícula de pessoas com deficiência na educação superior brasileira: subsídios normativos e ações institucionais para acesso e permanência. Revista IberoAmericana de Estudos em Educação, Araraquara, v. 10, n. esp., 2015, p. 643-60.

ARENHARDT, Daniel Luís; FRANCHI, Tatiane Stefanel; COSTA, Vânia Medianeira Flores; GROHMANN, Márcia Zampieri. Acessibilidade digital: uma análise em portais de Instituições Federais de Educação do Brasil. Arquivos Analíticos de Políticas Educativas, Rio de Janeiro, v. 25, n. 33, 2017, p. 1-28.

BACH, Catharine F; FERREIRA, Simone B. L; SILVEIRA, Denis. Avaliação de acessibilidade na web: estudo comparativo entre métodos de avaliação com a participação de deficientes visuais. ENCONTRO DA ANPAD, 33, 2009. Anais ... São Paulo: Anpad, 2009, p. 1-16.

BEM, Judite S; GIACOMINI, Nelci Maria Richter; WAISMANN, Moisés. Utilização da técnica da análise de clusters ao emprego da indústria criativa entre 2000 e 2010: estudo da Região do Consinos, RS. Interações, Campo Grande, v. 16, n. 1, 2015, p. 27-41.

BERGAMO, Fábio Vinicius de Macedo; PONCHIO, Mateus Canniatti; ZAMBALDI, Felipe; GIULIANI, Antonio Carlos; SPERS, Eduardo Eugênio. De Prospect a Aluno: Fatores Influenciadores da Escolha de uma Instituição de Ensino Superior. BASE - Revista de Administração e Contabilidade da Unisinos, São Leopoldo, v. 7, n. 3, 2010, p. 182-93.

BRASIL. Censo da educação superior 2018: notas estatísticas. Brasília: Inep, 2019. Disponível

http://download.inep.gov.br/educacao superior/censo superior/documentos/2019/censo da educacao superior 2018-notas estatisticas.pdf. Acesso em 7 mar. 2020.

BRASIL. Decreto n. 9.034, de 20 de abril de 2017: altera o decreto n. 7.824, de 11 de outubro de 2012, que regulamenta a lei n. 12.711, de 29 de agosto de 2012, que dispõe sobre o ingresso nas universidades federais e nas instituições federais de ensino técnico de nível médio. Brasília, 20 abr. 2017. Disponível em http://www.planalto.gov.br/ccivil 03/ Ato2015-2018/2017/Decreto/D9034.htm. Acesso em 06 mar. 2020.

BRASIL. Lei n. 13.146, de 6 de julho de 2015: institui a Lei Brasileira de Inclusão da Pessoa com Deficiência. Brasília, 6 jul. 2015. Disponível em http://www.planalto.gov.br/ccivil 03/ ato2015-2018/2015/lei/l13146.htm. Acesso em 26 mar. 2020.

BRASIL. Lei n. 12.711, de 29 de agosto de 2012: dispõe sobre o ingresso nas universidades federais e nas instituições federais de ensino técnico de nível médio e dá outras providências. Brasília, 29 ago. 2012. Disponível em http://www.planalto.gov.br/ccivil 03/ ato2011-2014/2012/lei/l12711.htm. Acesso em 06 mar. 2020. 
BRASIL. Decreto n. 7.611 , de 17 de novembro de 2011: dispõe sobre a educação especial, o atendimento educacional especializado e dá outras providências. Brasília, 17 nov. 2011a. Disponível em http://www.planalto.gov.br/ccivil 03/ Ato20112014/2011/Decreto/D7611.htm. Acesso em 07 mar. 2020.

BRASIL. Decreto n. 7.612, de 17 de novembro de 2011: institui o Plano Nacional dos Direitos da Pessoa com Deficiência - Plano Viver sem Limite. Brasília, 18 nov. 2011b. Disponível em http://www.planalto.gov.br/ccivil 03/ Ato20112014/2011/Decreto/D7612.htm. Acesso em 19 fev. 2020.

BRASIL. Edital n. 8, de 6 de julho de 2010: edital do Programa Incluir. Brasília, 7 jul. 2010. Disponível em http://portal.mec.gov.br/index.php?option=com docman\&view $=$ download\&alias=5809-edital-incluir-2010-dou\&category slug=junho-2010pdf\&ltemid=30192. Acesso em 19 fev. 2020.

BRASIL. Constituição da República Federativa do Brasil de 1988. Brasília, 5 out. 1988. Disponível em http://www.planalto.gov.br/ccivil 03/constituicao/constituicao.htm. Acesso em 20 fev. 2020.

CABRAL, Leonardo Santos Amâncio. Políticas de ações afirmativas, pessoas com deficiência e o reconhecimento das identidades e diferenças no ensino superior brasileiro. Arquivos Analíticos de Políticas Educativas, Rio de Janeiro, v. 26, n. 57, 2018, p. 1-33.

CAMPOS, Márcia de Borba; SANCHÉZ, Jaime; SOUZA, Thânia Clair de. Acessibilidade na web no Brasil: percepções de usuários com deficiência visual e de desenvolvedores web. CONGRESSO INTERNACIONAL DE INFORMÁTICA EDUCATIVA, 18, 2013. Anais... Porto Alegre: Tise, 2013, p. 325-333.

COSTA, Luana Ugalde da. Desenvolvimento profissional de coordenadores do Napne do Instituto Federal do Acre. Manaus: Ifam, 2018. 177f. Dissertação (Mestrado Profissional em Ensino Tecnológico). Instituto Federal de Educação, Ciência e Tecnologia do Amazonas.

CRESWELL, John. Projeto de pesquisa: métodos qualitativo, quantitativo e misto. Porto Alegre: Artmed, 2007.

FERREIRA, Gabriela Ayres; CIANCONI, Regina de Barros. Acessibilidade dos deficientes visuais e cegos às informações de bibliotecas universitárias na web. Informação \& Sociedade: Estudos, João Pessoa, v. 21, n. 2, 2011, p. 151-63.

FERREIRA, Simone B. L; SANTOS, Rodrigo C; SILVEIRA, Denis S. Panorama da acessibilidade na web brasileira. ENCONTRO DA ANPAD, 31, 2007. Anais... Rio de Janeiro: Anpad, 2007, p. 1-16.

FERREIRA, Simone B. L; CHAUVEL, Marie A; FERREIRA, Marcos G. A. L; Eacessibilidade: tornando visível o invisível. ENCONTRO DA ANPAD, 30, 2006. Anais... Salvador: Anpad, 2006, p. 1-17.

FREITAS, César; BRAGA, Mairene N; DELOU, Cristina M; CASTRO, H. A internet e os indivíduos com necessidades especiais: análise de acessibilidade de sites dos institutos federais no Brasil. Educação, Tecnologia e Cultura, Salvador, n. 13, 2016, p. 1-8.

GIL, Antônio Carlos. Métodos e técnicas de pesquisa social. São Paulo: Atlas, 2008.

GURGEL, Geovane Martine Moreira; MEDEIROS, Lucas Oliveira de; MOURA, Laisa Stephane Noronha Torres. Avaliação da acessibilidade do Portal IFRN à luz do E-MAG e do WCAG Samurai. Holos, Natal, v. 28, n. 1, 2012, p. 182-95. 
IBGE. Pesquisa nacional de saúde - 2013. Ciclos de vida: Brasil e grandes regiões. Rio de Janeiro: $\quad$ IBGE, $2015 . \quad$ Disponível em https://biblioteca.ibge.gov.br/visualizacao/livros/liv94522.pdf. Acesso em 19 fev. 2020.

LEMOS-SANTOS, Sylvana Karla da Silva de; TEIXEIRA, Laysse Noleto Balbino; KAFURE, Ivette. Acessível para quem? Uma análise da acessibilidade em sítios institucionais. Brazilian Journal of Development, Curitiba, v. 5, n. 6, 2019, p. 4976-89.

LOPES, Soraya Tatyara Costa; MARTINS, Chicar Fabiane Silva; CHAVES, Adriana Alves da Silva; TAVARES, Ana Carolina Cerveira. Educação profissional e tecnológica: a acessibilidade como garantia de inclusão a alunos com deficiência. Revista Eixo, Brasília, v. 5, n. 2, 2016, p. 10-20.

MACIEL, Carina Elisabeth; ANACHE, Alexandra Ayach. A permanência de estudantes com deficiência nas universidades brasileiras. Educar em Revista, Curitiba, v. 33, n. esp., 2017, p. 71-86.

MARCONI, Marina A; LAKATOS, Eva M. Fundamentos de metodologia científica. São Paulo: Atlas, 2003, p. 174-214.

MELO, Francisco Ricardo Lins Vieira de; ARAÚJO, Eliana Rodrigues. Núcleos de acessibilidade nas universidades: reflexões a partir de uma experiência institucional. Psicologia Escolar e Educacional, São Paulo, n. esp., 2018, p. 57-66.

MELO, Francisco V. S; SILVEIRA, Denis S; FERREIRA, Simone B. L. Os cegos conseguem enxergar destinos turísticos na internet? Uma análise da acessibilidade de websites oficiais dos estados brasileiros. ENCONTRO DA ANPAD, 37, 2013. Anais... Rio de Janeiro: Anpad, 2013, p. 1-15.

MENDES, Katiuscia Aparecida Moreira de Oliveira. Educação especial inclusiva nos institutos federais de educação, ciência e tecnologia brasileiros. Goiânia: UFG, 2017. 168f. Tese (Doutorado em Educação). Programa de Pós-Graduação em Educação, Universidade Federal de Goiás.

NOGUEIRA, Lilian de Fátima Zanoni; OLIVER, Fátima Corrêa. Núcleos de acessibilidade em instituições federais brasileiras e as contribuições de terapeutas ocupacionais para a inclusão de pessoas com deficiência no ensino superior. Cad. Bras. Ter. Ocup., São Carlos, v. 26, n. 4, 2018, p. 859-82.

PEREIRA, Marilú Mourão. Ações afirmativas e a inclusão de alunos com deficiência no Ensino Superior. Ponto de Vista, Florianópolis, n. 10, 2008, p. 19-38.

ROCHA, Vânia Meneghini da. A educação especial nos institutos federais: o que dizem os planos de desenvolvimento institucionais? Joinvile: Univille, 2016. 162f. Dissertação (Mestrado em Educação). Programa de Mestrado em Educação, Universidade da Região de Joinvile.

SANTOS, Evelyn; GONÇALVES, Manuela; RAMOS, Isabel; CASTRO, Lisneti; LOMEO, Roselane. Inclusão no ensino superior: percepções dos estudantes com necessidades educativas especiais sobre o ingresso à universidade. Revista Portuguesa de Educação, Braga, v. 28, n. 2, 2015, p. 251-70.

TORRES, Elisabeth Fátima; MAZZONI, Alberto Angel; ALVES, João Bosco da Mota. A acessibilidade à informação no espaço digital. Ciência da Informação, Brasília, v. 31, n. 3, 2002, p. 83-91.

UFCG. Resolução n. 11/2016, de 31 de maio de 2016: cria o Núcleo de Acessibilidade e Inclusão. Campina Grande: UFCG, 2016. Disponível em http://www.ufcg.edu.br/ costa/resolucoes/res 12112016.pdf. Acesso em 7 abr. 2020. 
UFRN. Resolução n. 193/2010-CONSEPE, de 21 de setembro de 2010: dispõe sobre o atendimento educacional a estudantes com necessidades educacionais especiais na Universidade Federal do Rio Grande do Norte. Natal: UFRN, 2010. Disponível em https://sigrh.ufrn.br/sigrh/public/colegiados/filtro busca.jsf. Acesso em 17 mar. 2020.

WORLD WIDE WEB CONSORTIUM [W3C]. Diretrizes de acessibilidade para conteúdo web (WCAG) 2.0. Disponível em https://www.w3.org/Translations/WCAG20-ptPT/WCAG20-pt-PT-20141024/. Acesso em 19 mar. 2020.

Thales Fabricio da Costa e Silva é psicólogo na Universidade Federal de Campina Grande.

Orcid: https://orcid.org/0000-0003-1828-3259.

Endereço: Rua Bernardino Ferreira, 651 - 59987-000 - Riacho de Santana - RN Brasil.

E-mail: thalespsic@gmail.com.

Luana Ugalde da Costa é professora de Psicologia no Instituto Federal de Educação, Ciência e Tecnologia do Acre.

Orcid: https://orcid.org/0000-0001-5332-8956.

Endereço: Rod. AC 10, 2095, Alto Alegre - 69921-282 - Rio Branco - AC - Brasil.

E-mail: luana.costa@ifac.edu.br.

Débora Bruna Alves Almeida é administradora no Instituto Federal de Educação, Ciência e Tecnologia do Ceará.

Orcid: https://orcid.org/0000-0002-9758-3227.

Endereço: Rua Manoel Saraiva, 1494 - 62930-000 - Limoeiro do Norte - CE - Brasil.

E-mail: debora.almeida@ifce.edu.br.

Leandro Campi Prearo é professor no Programa de Pós-Graduação em Administração da Universidade Municipal de São Caetano do Sul.

Orcid: https://orcid.org/0000-0002-6039-1280.

Endereço: Rua Conceição, 321 - 09530-060 - São Caetano do Sul - SP - Brasil.

E-mail: leandro.prearo@gmail.com.

Recebido em 10 de junho de 2020.

Aceito em 2 de outubro de 2020.

(c) $(1) \otimes(\Theta$ 